\section{Deutsche Ausgabe der PRO-CTCAE validiert}

Die Perspektive des Patienten wird bei der Erfassung unerwünschter Ereignisse wichtiger. Daher hat das National Cancer Institute (NCI) 2011 den Kriterien zur Erfassung unerwünschter Ereignisse (CTCAE: Common Terminology Criteria for Adverse Events) eine Version mit den „patient reported outcomes" zur Seite gestellt (PRO-CTCAE). Dafür liegt nun eine deutsche Übersetzung vor.

D ie Versionen der CTCAE für Ärzte und Patienten ergänzen sich. Die deutsche Übersetzung wurde bereits linguistisch geprüft, eine quantitative Validierung stand bisher aus. Dafür wurden 31 der insgesamt 124 Items als Kerngruppe ausgewählt, die 14 Nebenwirkungen abbilden und bei verschiedensten Tumorlokalisationen und -therapien von Bedeutung sind.

Dieser Fragenkatalog wurde gemeinsam mit dem EORTC-Questionnaire zur Lebensqualität (EORTC-QLQ-C30) sowie dem OMDQ (Oral Mucositis Daily Questionnaire) bei Patienten mit unterschiedlichen Tumoren an 10 Tumorzentren in Deutschland und Österreich verteilt.

Von 660 verteilten Katalogen wurden 271 zurückgesendet (41\%), und 262 davon waren auswertbar. Die Analyse der Item-Qualität sprach für die Verständlichkeit der Fragen. Die Zuverlässigkeit (reliability) war sehr gut; der Cronbach's alpha Korrelationskoeffizient betrug $>0,9$ für fast alle Item-Cluster. Die Konstrukt-Validität der 31 PROCTCAE-Items wurde bestätigt und es konnten 10 inhaltlich wichtige ItemCluster mittels PCA („principal compo- nent analysis") identifiziert werden. Darüber hinaus wurde die KonstruktValidität auch anhand des MTMMVerfahrens („multitrait-multimethod matrix“) überprüft. Die PRO-CTCAEScores fielen bei Patienten mit verminderter Lebensqualität signifikant niedriger aus als bei solchen mit erhaltener Lebensqualität.

Fazit: Die Erfassung der Symptome und Nebenwirkungen mittels PatientenSelbsturteil (PRO-CTCAE) sollte parallel zur CTCAE-Dokumentation durch Ärzte vorgenommen werden. PRO-CTCAE liefert zusätzliche Informationen, die die bisher bestehende Erhebung von Sicherheit und Verträglichkeit einer Tumortherapie komplementär ergänzen können. Brigitte Schalhorn

Hagelstein V et al. Validation of the German patient-reported outcomes version of the common terminology criteria for adverse events (PRO-CTCAE ${ }^{\mathrm{TM}}$ ). Ann Oncol. 2016;27(12):2294-9.

\title{
Pneumonitis unter PD1-/PD-L1-Therapie
}

\begin{abstract}
Antikörper gegen PD-1/PD-L1 haben sich als echter Fortschritt in der Therapie verschiedener solider Tumoren erwiesen. Die Pneumonitis ist eine nicht sehr häufige, aber potenziell lebensbedrohliche Toxizität dieser Therapie, die bislang nur in Fallberichten und kleineren Serien beschrieben wurde.
\end{abstract}

es sich um die Schweregrade 1 und 2, Grad 3 trat bei $23 \%$ und die Grade 4 und 5 bei je einem Patienten auf. Bei leichter Pneumonitis (Grad 1; $\mathrm{n}=17$ ) reichte meist der Therapieabbruch zur völligen Normalisierung, Steroide waren nur bei 2 Patienten nötig. Bei Grad 2 erhielten alle Patienten Steroide, teils sogar intravenös. Bei allen nachbeobachteten 13 Patienten kam es zu einer Besserung, bei 10 zur völligen Normalisierung. 5 der 12 Patienten mit Pneumonitis vom Grad 3 erhielten zu den Steroiden eine Immunsuppression. Eine Besserung oder Heilung trat bei 6 ein, 4 verschlechterten sich jedoch weiter. Insgesamt ereigneten sich 5 Todesfälle, 3 durch Infektionen nach lange dauernder Immunsuppression.

Fazit: Es ist wichtig, an eine Pneumonitis als Komplikation zu denken, um sie

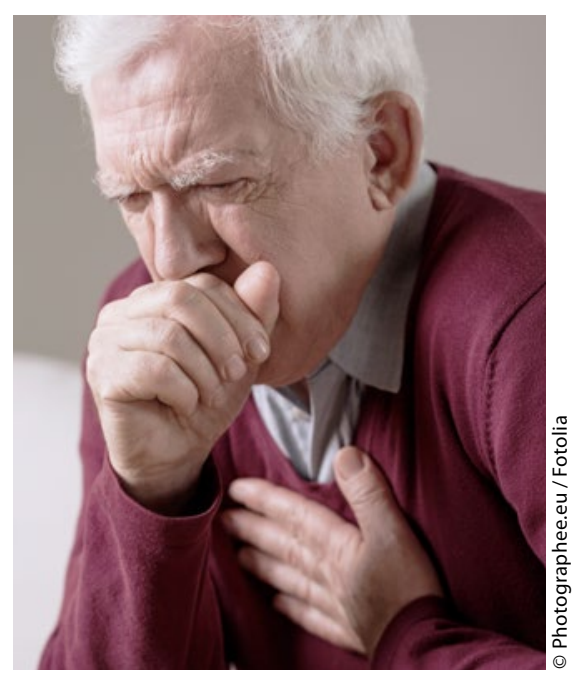

Husten war nach Dyspnoe das zweithäufigste Symptom der Pneumonitis.

frühzeitig zu behandeln. Leichtere Formen bessern sich nach Therapieabbruch oder unter Steroiden rasch, aber es gibt auch komplizierte und sogar lebensbedrohliche Verläufe. Brigitte Schalhorn

Naidoo J et al. Pneumonitis in patients treated with anti-programmed death-1/programmed death-ligand 1 therapy. J Clin Oncol. 2017;35(7):709-717. 